\title{
Síntesis, caracterización y evaluación de la resistencia a la corrosión de recubrimientos híbridos Sol-Gel base TEOS/MPS sobre la aleación AA2050-T8
}

\author{
Claudia Nieves ${ }^{\mathrm{a}}$, Erika N. Remolina ${ }^{\mathrm{a}}$, Carlos A. Hernándeza ${ }^{\mathrm{a}}$ Laura M. Rueda ${ }^{\mathrm{a}}$, Ana E. Coya \\ Fernando Viejo ${ }^{\mathrm{b}, \varpi}$ \\ ${ }^{a}$ Escuela de Ingeniería Metalúrgica y Ciencia de Materiales, Universidad Industrial de Santander, Calle 9 \#27, Bucaramanga, \\ Santander, Colombia

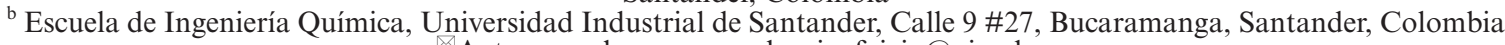 \\ Autor para la correspondencia: fviejo@uis.edu.co
}

Enviado: 10 Diciembre 2016; Aceptado: 14 Mayo 2017; Publicado on-Line: 27 Noviembre 2017

RESUMEN: El presente trabajo tuvo como objetivo sintetizar, caracterizar y evaluar la resistencia a la corrosión de recubrimientos híbridos sol-gel base TEOS/MPS, sintetizados sobre la aleación AA2050-T8 (Al-Cu-Li) bajo diferentes condiciones experimentales de polimerización, tales como solvente y tiempo de envejecimiento, así como diferentes relaciones molares de los precursores inorgánico tetraetoxisilano (TEOS) e híbrido 3-metacriloxipropiltrimetoxisilano (MPS). Se monitoreó la evolución de los soles durante el proceso de envejecimiento mediante espectroscopía infrarroja (FT-IR) y ensayos reológicos. Los recubrimientos se obtuvieron mediante dip-coating y fueron caracterizados a través de microscopía electrónica de barrido (MEB-EDX). La evaluación de la resistencia a la corrosión se realizó mediante ensayos de polarización anódica potenciodinámica, en solución aireada $0,1 \mathrm{M}$ de $\mathrm{NaCl}$. Los resultados obtenidos revelaron que, de los tres solventes evaluados, el etanol es el que permite mejor estabilización del precursor híbrido y favorece el proceso de polimerización de los grupos metacrilato del precursor MPS. Por otra parte, la variación de la relación molar TEOS:MPS conlleva a la obtención de recubrimientos más homogéneos y continuos al incrementar la relación del precursor inorgánico. Finalmente, todos los recubrimientos sintetizados mejoraron la resistencia a la corrosión de la aleación AA2050-T8, en especial los sintetizados bajo la relación TEOS:MPS 80:20.

PALABRAS CLAVE: Aluminio; Corrosión; Recubrimientos; Sol-Gel

Citar como/Citation: Nieves, C.; Remolina, E.N.; Hernández, C.A.; Rueda, L.M.; Coy, A.E.; Viejo, F. (2017) "Síntesis, caracterización y evaluación de la resistencia a la corrosión de recubrimientos híbridos Sol-Gel base TEOS/MPS sobre la aleación AA2050-T8”. Rev. Metal. 53(4): e106. http://dx.doi.org/10.3989/revmetalm.106

\begin{abstract}
Synthesis, characterisation and corrosion resistance of TEOS/MPS hybrid sol-gel coatings deposited on the AA2050-T8 alloy. The aim of the present work was to synthesize, characterise and evaluate the corrosion resistance of TEOS/MPS hybrid sol-gel coatings applied on the AA2050-T8 aluminium alloy attending to two experimental variables: the sol synthesis conditions (type of solvent and aging time), and the inorganic/hybrid (TEOS/MPS) ratio. The aging process was monitored by infrared spectroscopy (FTIR) and viscosity measurements. The hybrid coatings were obtained by dip-coating and then characterized by scanning electron microscopy (SEM-EDX). The evaluation of corrosion resistance was performed by anodic potentio dynamic polarization testing in aerated $0.1 \mathrm{M} \mathrm{NaCl}$ solution. The results showed that ethanol as solvent promoted better stabilization of the hybrid precursor and favoured polymerization of the
\end{abstract}


methacrylate groups of the MPS precursor. Further, an increase of the TEOS/MPS ratio allowed obtaining more continuous and uniform coatings. Nevertheless, all the hybrid coatings showed a significant increase in corrosion resistance of the AA2050-T8 alloy, being more evident for those synthesized with the TEOS:MPS $80: 20$ ratio.

KEYWORDS: Aluminium; Coating; Corrosion; Sol-Gel

ORCID ID: Claudia Nieves (http://orcid.org/0000-0002-4174-1572); Erika N. Remolina (http://orcid.org/00000002-5164-7408); Carlos A. Hernández (http://orcid.org/0000-0002-5857-1039); Laura M. Rueda (http://orcid. org/0000-0002-6664-7611); Ana E. Coy (http://orcid.org/0000-0001-8813-0042); Fernando Viejo (http://orcid. org/0000-0001-7628-8213)

Copyright: ( 2017 CSIC. Este es un artículo de acceso abierto distribuido bajo los términos de la licencia Creative Commons Attribution (CC BY) España 3.0.

\section{INTRODUCCIÓN}

Las aleaciones de aluminio han sido ampliamente estudiadas por la industria aeronáutica debido a su excelente relación resistencia/peso y su buena tolerancia al daño (Polmear, 2005). Dentro de las aleaciones de aluminio más utilizadas en dicha industria se encuentran las pertenecientes a la serie AA7 $x x x(\mathrm{Al}-\mathrm{Zn}-\mathrm{Mg})$, que exhiben la mayor resistencia mecánica de todas las aleaciones de aluminio; sin embargo, presentan baja tolerancia al daño (Polmear, 2005; Viejo et al., 2010a). Por otro lado, se encuentran las aleaciones de la serie AA2xxx (Al-Cu) que presentan destacada tolerancia al daño y estabilidad térmica, pero con una menor resistencia mecánica comparada con las aleaciones de la serie AA7xxx (Li et al., 2005; Viejo et al., 2010b).

Actualmente, existe un creciente interés por las aleaciones $\mathrm{Al}-\mathrm{Cu}-\mathrm{Li}$, pertenecientes a la serie AA2xxx, denominadas aleaciones de tercera generación, ya que presentan una resistencia mecánica comparable con las aleaciones AA7 xxx, combinada con la excelente tolerancia al daño de las aleaciones AA2xxx. Así mismo, la presencia de Li disminuye el peso de la aleación en aproximadamente $3 \%$ en comparación con las otras aleaciones de aluminio (Crill et al., 2006; Warner, 2006; Li et al., 2008; Guérin et $a l ., 2015)$. Dentro de este grupo de aleaciones, se destaca la aleación AA2050-T8 que, debido a su excelente combinación de resistencia, densidad, resistencia a la fatiga, estabilidad térmica y tenacidad, se ha convertido en candidata atractiva en aplicaciones aeronáuticas que requieren alta resistencia específica y excelente tolerancia al daño (Lequeu et al., 2010; Malard et al., 2015; Li et al., 2016).

No obstante, al alear el aluminio con otros elementos para aumentar las propiedades mecánicas, se origina una reducción en la resistencia a la corrosión principalmente en ambientes marinos y/o húmedos que viene determinada por la concentración y distribución de dichos elementos
(Li et al., 2007; Li et al., 2008; Viejo et al., 2010a). Particularmente, para las aleaciones $\mathrm{Al}-\mathrm{Cu}-\mathrm{Li}$ tales como la AA2050-T8, la presencia de cobre, hierro y manganeso dan lugar a la formación de fases intermetálicas de naturaleza catódica que originan problemas de corrosión localizada tanto por picadura como galvánica; mientras que, las fases intermetálicas ricas en cobre y litio dan lugar a fases de naturaleza anódica provocando problemas de corrosión por exfoliación e intergranular (Viejo et al., 2010a; Viejo et al., 2010b; Boag et al., 2011; Gharbi et al., 2017).

Dentro de los métodos de protección para las aleaciones de aluminio existen diversas técnicas de las que son comúnmente estudiadas el anodizado, la conversión química, la modificación superficial con láser, entre otras (Liu et al., 2008; Zheng y Li, 2010; Coy et al., 2010; Ma et al., 2011; Embuka et al., 2017). Dentro de dichas técnicas, recientemente se ha prestado interés al método sol-gel, que permite la obtención de recubrimientos cerámicos que actúan como barreras físicas frente a los procesos de corrosión y desgaste, empleando un equipamiento relativamente sencillo y con posibilidad de aplicación sobre sustratos con formas complejas (Wang y Bierwagen, 2009; Zheng y Li, 2010).

Para la síntesis de recubrimientos vía sol-gel se dispone de precursores de recubrimientos inorgánicos e híbridos. Los precursores comúnmente utilizados para la síntesis de recubrimientos inorgánicos son los alcóxidos metálicos de tipo $\mathrm{M}(\mathrm{OR})$ n, siendo $\mathrm{M}$ un metal como $\mathrm{Si}, \mathrm{Ti}, \mathrm{Zr}, \mathrm{Al}, \mathrm{Fe}, \mathrm{B}$, entre otros, aunque normalmente suelen ser base silano $\left(\mathrm{Si}(\mathrm{OR})_{4}\right)$, por su reactividad moderada que hace más controlable el proceso de síntesis (Han et al., 2007; Wang y Bierwagen, 2009; Zheng y Li, 2010). Entre ellos aparece el tetraetoxisilano $\left(\mathrm{Si}(\mathrm{OEt})_{4}, \mathrm{TEOS}\right)$, convencionalmente utilizado en la síntesis de estructuras de sílice $\left(\mathrm{SiO}_{2}\right)$ para catalizadores, membranas y fármacos (Bonekamp et al., 2008). Estos recubrimientos se destacan por su excelente adhesión al sustrato metálico; no obstante, presentan problemas para obtener 
espesores superiores a un micrómetro $(1 \mu \mathrm{m}) \sin$ que exista agrietamiento durante el proceso de secado (Wang y Bierwagen, 2009; Zheng y Li, 2010).

Por otro lado, los precursores de recubrimientos híbridos presentan estructura $\mathrm{R}^{\prime} \mathrm{M}(\mathrm{OR})_{\mathrm{n}-1}$, donde un grupo alcóxido (RO) es sustituido por un grupo funcional orgánico R', tipo fenilo, epoxi, vinilo, metacrilato, amino, etc. (Hofacker et al., 2002; Wang y Bierwagen, 2009; HernándezBarrios et al., 2014; Remolina et al., 2017). Dentro estos precursores híbridos, el 3-metacriloxipropiltrimetoxisilano (MPS), cuyo grupo funcional es del tipo metacrilato, es un precursor ampliamente utilizado ya que permite la modificación de la funcionalidad de las películas. Sin embargo, la presencia de este tipo de precursores puede llegar a disminuir la adhesión recubrimiento-metal (Guo et al., 2006; Rosero et al., 2010a; Wang et al., 2010).

Es por ello que, en la actualidad, se está proponiendo la síntesis de recubrimientos a partir de la combinación de ambos tipos de precursores para dar lugar a un nuevo tipo de recubrimientos híbridos en los cuales, el precursor inorgánico (usualmente TEOS) contribuye a incrementar la adhesión entre el recubrimiento y el metal (Rueda et al., 2016); mientras que el componente híbrido (como el MPS), aumenta la flexibilidad y la densidad, reduce la tendencia al agrietamiento, y el tiempo y la temperatura de curado de las películas incluso hasta temperatura ambiente, resultando apropiado para las aleaciones de aluminio que no pueden ser tratadas a elevada temperatura (Bonekamp et al., 2008; Wang y Bierwagen, 2009; Zheng y Li, 2010; Remolina et al., 2017).

Pese a lo anterior, las condiciones de síntesis que incluyen la polimerización del MPS aún no han sido estudiadas en detalle, ya que las que se conocen son para monómeros convencionales y no para precursores base silano (Moulay, 2010). Con base en las anteriores premisas, el objetivo del presente trabajo fue sintetizar, caracterizar y evaluar el desempeño frente a la corrosión de recubrimientos híbridos sol-gel base TEOS/MPS depositados sobre la aleación AA2050-T8 (Al-Cu-Li) atendiendo a diferentes condiciones experimentales de síntesis (solvente, tiempo de envejecimiento, relación molar de precursores).

\section{MATERIALES Y MÉTODOS}

\section{a. Material objeto de estudio}

El material objeto de estudio fue la aleación AA2050-T8 (Al-Cu-Li), proporcionada bajo tratamiento térmico T8 (TT de solución, deformación y posterior envejecimiento artificial) por la compañía Alcan Aerospace (Reino Unido). La composición nominal de la aleación se muestra en la Tabla 1.

\section{b. Síntesis y caracterización de los soles híbridos}

Para la síntesis de los soles se empleó tetraetoxisilano (TEOS, Alfa Aesar, 98\%) como precursor inorgánico y 3-metacriloxipropil-trimetoxisilano (MPS, Aldrich, 98\%) como precursor híbrido; como iniciador del proceso de polimerización se utilizó peróxido de benzoílo en una relación de $0,01 \mathrm{~mol} / \mathrm{mol}$ de dobles enlaces $\mathrm{C}=\mathrm{C}$ (Rosero et al., 2010a; Ohtsuki et al., 2002). La caracterización de los soles se desarrolló en dos etapas. En la primera etapa se evaluó la influencia del tipo de solvente en el proceso de polimerización del precursor MPS. Para ello fueron utilizados tres solventes diferentes: tolueno como solvente apolar-aprótico, tetrahidrofurano como solvente polar-aprótico y etanol como solvente polar-prótico (Barry Carte y Grant Norton, 2007). Los soles fueron sintetizados empleando el precursor híbrido, el iniciador de la polimerización y un solvente en una relación en volumen de 1:1 de solvente con respecto al precursor MPS (Rosero et al., 2009; Rosero et al., 2010b).

Una vez seleccionado el solvente, en una segunda etapa fue evaluada la influencia del tiempo de envejecimiento del proceso de hidrólisis y condensación del sol híbrido por un tiempo de hasta 7 días. Para ello, se incluyó TEOS, $\mathrm{HNO}_{3}(0,6 \%$ vol.) como catalizador de las reacciones de hidrólisis y condensación, y se mantuvo la relación volumen de 1:1 de solvente, esta vez con respecto a la mezcla de precursores (Contreras et al., 2015). Finalmente, se evaluó la influencia de diferentes relaciones molares TEOS:MPS (80:20, 60:40: 40:60, 20:80). El pH de los soles para todas las relaciones molares se fijó en un valor de 3 .

Los soles fueron monitoreados mediante espectroscopía infrarroja (FT-IR) y ensayos reológicos a temperatura de $25^{\circ} \mathrm{C}$. Para ello se empleó un

TABLA 1. Composición nominal de la aleación AA2050-T8 (Al-Cu-Li) (\% peso)

\begin{tabular}{lccccccccccc}
\hline \% peso & $\mathbf{C u}$ & $\mathbf{L i}$ & $\mathbf{M g}$ & $\mathbf{M n}$ & $\mathbf{A g}$ & $\mathbf{F e}$ & $\mathbf{T i}$ & $\mathbf{S i}$ & $\mathbf{Z r}$ & $\mathbf{O T R O S}$ & $\mathbf{A l}$ \\
\hline Min & 3,2 & 0,7 & 0,2 & 0,2 & 0,2 & - & - & - & 0,06 & - & \multirow{2}{*}{ Balance } \\
Max & 3,9 & 1,3 & 0,6 & 0,5 & 0,8 & 0,10 & 0,1 & 0,08 & 0,14 & 0,20 & \\
\hline
\end{tabular}


espectrofotómetro infrarrojo con transformada de Fourier (FT-IR) SHIMADSU FTIR-84005 y un reómetro digital BROOKFIELD modelo DV.III + RHEOMETER V5.0 con una aguja ULA 0.0 a $240 \mathrm{rpm}$.

\section{c. Deposición de los recubrimientos híbridos}

La deposición de los recubrimientos se llevó a cabo mediante la técnica de dip-coating, para lo cual fueron preparadas superficialmente muestras de geometría rectangular con dimensiones de $15 \times 10 \times 5 \mathrm{~mm}$ mediante desbaste gradual con papel de carburo de silicio, $\mathrm{SiC}$, hasta granulometría P1200. A continuación, las muestras fueron sumergidas verticalmente en el sol a una velocidad constante de $6 \mathrm{~cm} \cdot \mathrm{min}^{-1}$ donde permanecieron por 2 min, con el fin de garantizar la mojabilidad del sol sobre la superficie de la muestra. Transcurrido dicho tiempo, las muestras se extrajeron a la misma velocidad empleada para la inmersión y, posteriormente, se sometieron a un tratamiento térmico de curado que consistió en $30 \mathrm{~min}$ a temperatura ambiente y, a continuación, $2 \mathrm{~h}$ a $60^{\circ} \mathrm{C}$ y $2 \mathrm{~h}$ más a $120^{\circ} \mathrm{C}$.

\section{d. Caracterización de los recubrimientos híbridos}

Con el fin de estudiar características superficiales tales como continuidad, espesor, uniformidad, composición y rugosidad, los diferentes recubrimientos obtenidos fueron examinados mediante microscopía electrónica de barrido (MEB). Para ello se empleó un microscopio electrónico de barrido ambiental (MEB-EDX) QUANTA FEG 650 que presenta sistemas de detección de energías dispersivas de Rayos $\mathrm{X}(\mathrm{EDX})$ y análisis de imagen de electrones retrodispersados (BSE).

\section{e. Evaluación de la resistencia a la corrosión}

Con objeto de evaluar la resistencia a la corrosión que ofrecen los recubrimientos obtenidos bajo diferentes relaciones molares TEOS:MPS y tiempos de envejecimiento, se llevaron a cabo ensayos electroquímicos de polarización anódica potenciodinámica, mediante el uso de un potenciostato GAMRY 6000. Se diseñó una celda electroquímica de tres electrodos, compuesta de un electrodo auxiliar (platino), un electrodo de referencia (calomelanos saturados) y un electrodo de trabajo, en este caso la muestra objeto de estudio. El medio de ensayo utilizado fue una solución aireada de $0,1 \mathrm{M}$ de $\mathrm{NaCl}$, mientras que el área de exposición de las muestras fue de $1 \mathrm{~cm}^{2}$.

Las muestras se sumergieron previamente en la solución salina por $1 \mathrm{~h}$ con objeto de permitir la estabilización del sistema. Una vez alcanzado dicho tiempo, se procedió a realizar el ensayo de polarización anódica en el que se llevó a cabo un barrido anódico de potencial desde $-100 \mathrm{mV}$ hasta $2000 \mathrm{mV}$ (con respecto al potencial de circuito abierto (OCP)), con una velocidad de barrido de $0,3 \mathrm{mV} \cdot \mathrm{s}^{-1}$ y una densidad de corriente límite de $1 \mathrm{~mA} \cdot \mathrm{cm}^{-2}$.

\section{RESULTADOS Y DISCUSIÓN}

\subsection{Caracterización de los soles híbridos}

En la Fig. 1 se presentan los espectros FT-IR para la región comprendida entre $1800-1600 \mathrm{~cm}^{-1}$, donde aparecen los modos de vibración representativos de los enlaces $\mathrm{C}=\mathrm{O}$ y $\mathrm{C}=\mathrm{C}$ relacionados con el proceso de estabilización y polimerización respectivamente del MPS en los soles. En general, se observa que los espectros correspondientes a los soles sintetizados con tolueno (Fig. 1a) y tetrahidrofurano (Fig. 1b) como solventes no se ven alterados con el transcurso del tiempo. Por otro lado, el espectro correspondiente al sol empleando etanol (Fig. 1c) sí presenta una evolución, observándose un ensanchamiento de las bandas en la zona estudiada debido a dos razones: i) en la región entre $1735-1680 \mathrm{~cm}^{-1}$ la banda relacionada con las vibraciones por tensión de los enlaces $\mathrm{C}=\mathrm{O}$ ubicada inicialmente en $1720 \mathrm{~cm}^{-1}$ presenta un ensanchamiento probablemente debido a la formación de puentes de hidrógeno alrededor de $1700 \mathrm{~cm}^{-1}$ entre el grupo carbonilo y el etanol, favoreciendo la estabilización del precursor híbrido (Rosero et al., $2010 \mathrm{~b}$ ); y ii) en la región entre $1650-1620 \mathrm{~cm}^{-1}$, la banda asociada con las vibraciones por tensión de los enlaces $\mathrm{C}=\mathrm{C}$ ubicada inicialmente en $1638 \mathrm{~cm}^{-1}$ presenta un ensanchamiento relacionado probablemente con el progreso del proceso de polimerización del precursor híbrido al romperse el doble enlace $\mathrm{C}=\mathrm{C}$.

Por otra parte, en las Figs. 2a y $2 \mathrm{~b}$ se presenta el análisis por deconvolución de los espectros FTIR correspondientes a los precursores TEOS y MPS en la región comprendida entre 1250 y $1000 \mathrm{~cm}^{-1}$ relacionada con las reacciones de hidrólisis y condensación presentes en el proceso sol-gel.

Para el espectro infrarrojo del TEOS (Fig. 2a) se identificaron 3 bandas a 1170,1110 y $1080 \mathrm{~cm}^{-1}$ asociadas a las vibraciones de los enlaces Si-O-C (Téllez et al., 2004; Wang et al., 2010); mientras que, para el precursor MPS, además de las bandas ya mencionadas, se detectó la presencia de las bandas a $1200,1190 \mathrm{~cm}^{-1}$ originadas por las vibraciones de los enlaces $\mathrm{Si}-\mathrm{C}$ y a $1040 \mathrm{~cm}^{-1}$ relacionada con las vibraciones por tensión de los enlaces C-O, todas relacionadas con la cadena orgánica (Limpo et al., 1993; Peña et al., 2007; Criado et al., 2014), (Fig. 2b).

En cuanto a la evolución de las reacciones de hidrólisis y condensación de los soles híbridos, en 

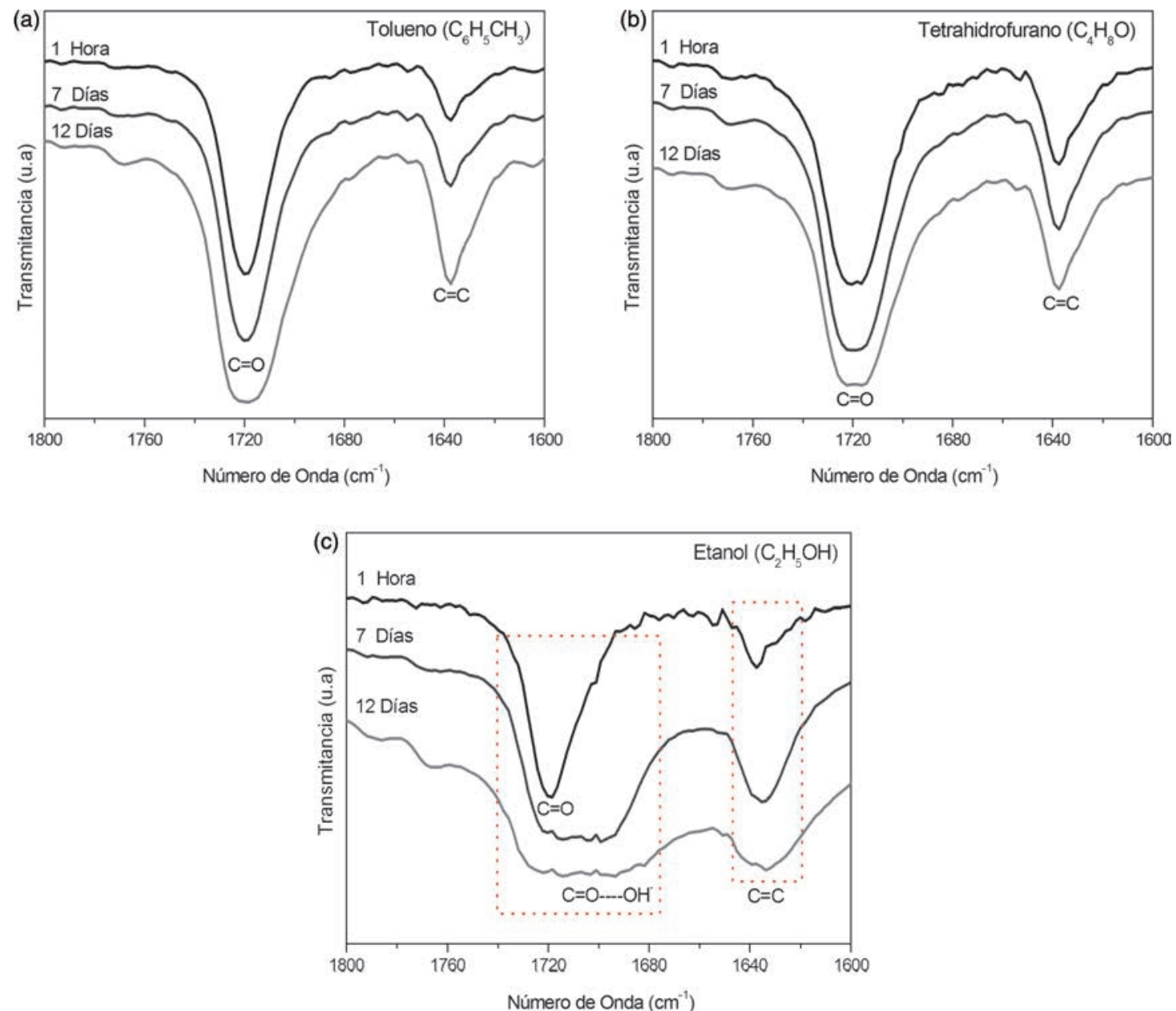

FIGURA 1. Espectros FT-IR de los soles para la relación TEOS:MPS 60:40 preparados con diferentes solventes.

las Figs. 2c y 2d se presenta el análisis por deconvolución de los espectros IR de los soles a 1 hora y 7 días de envejecimiento, respectivamente. En este sentido, se evidencia la disminución progresiva de la intensidad relativa de las bandas relacionadas con las vibraciones Si-O-C a 1170,1110 y $1080 \mathrm{~cm}^{-1}$, lo que se atribuye a la evolución de las reacciones de hidrólisis de los precursores. Así mismo, debido al avance de las reacciones de condensación se aparecen dos nuevas bandas a 1055 y $1135 \mathrm{~cm}^{-1}$ asociadas a las vibraciones de los enlaces Si-O-Si (Jiang et al., 2008; Criado et al., 2014).

Adicionalmente, la evolución de la viscosidad de los soles para diferente relaciones molares TEOS:MPS se presenta en la Fig. 3, en donde se observa un aumento gradual de la viscosidad de los soles con el incremento del precursor híbrido, pasando de $2,35 \mathrm{cP}$ a $2,95 \mathrm{cP}$ (un incremento de alrededor del $25 \%$ ) para relaciones molares de 80:20 a 20:80, respectivamente, después de 6 horas de envejecimiento de los soles. El comportamiento de los soles ante el incremento del precursor híbrido se relaciona probablemente con la mayor proporción de enlaces $\mathrm{C}=\mathrm{C}$ susceptibles de reaccionar y aumentar el grado de entrecruzamiento en el sol, así como al mayor grado de viscosidad del precursor MPS con relación al precursor inorgánico TEOS.

\subsection{Caracterización de los recubrimientos}

A modo representativo en la Fig. 4 se presentan las micrografías electrónicas de los recubrimientos sintetizados sobre la aleación de aluminio AA2050-T8 para la relación molar 60:40 TEOS:MPS y diferentes tiempos de envejecimiento.

En general, a medida que incrementa el tiempo de envejecimiento del sol, se obtienen recubrimientos más continuos y uniformes. Este hecho está asociado al mayor grado de hidrólisis del sol previo al momento de la deposición de los 

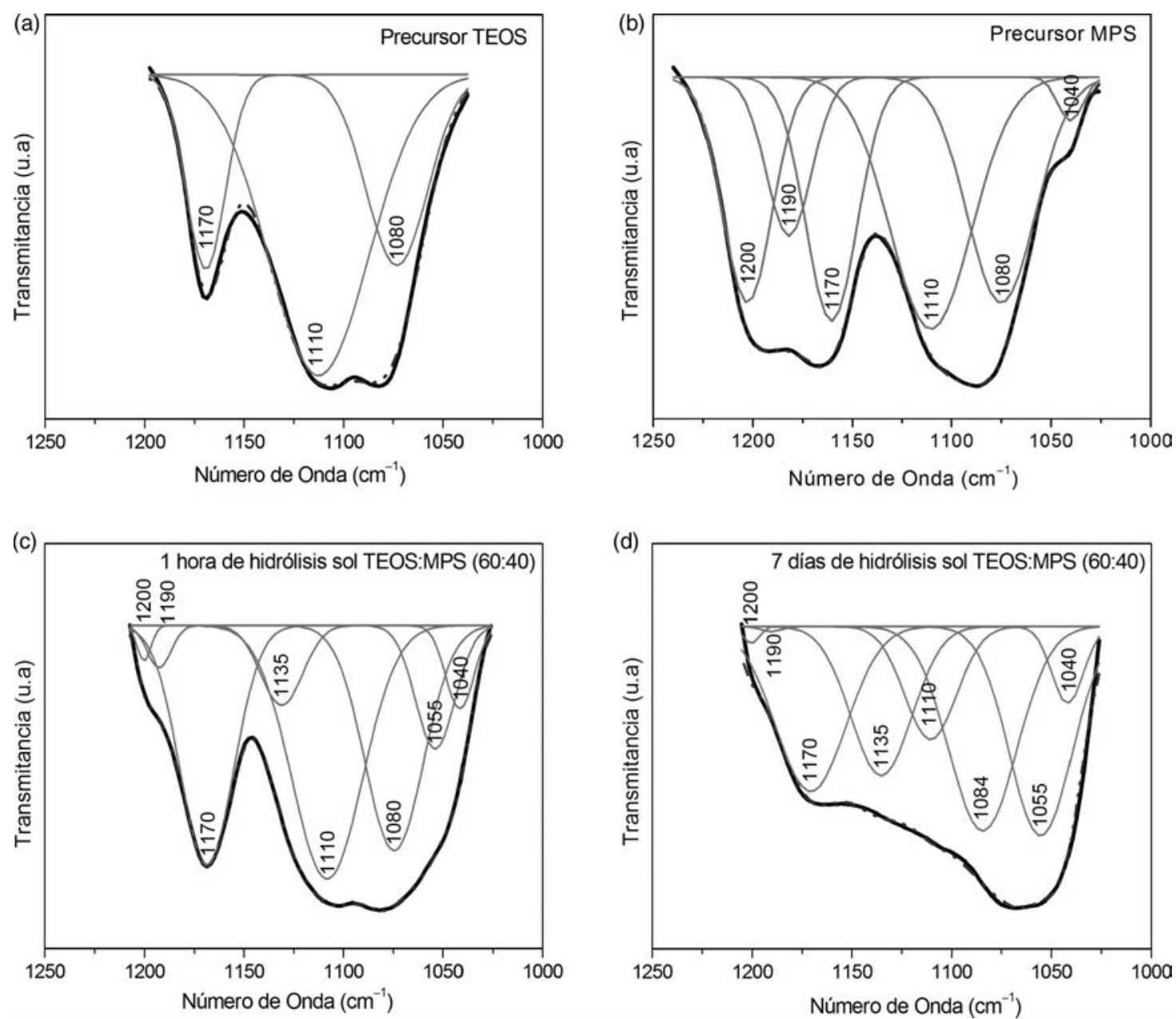

FIgURA 2. Espectros FTIR de: a) MPS; b) TEOS; proceso hidrólisis y condensación a: c) 1 hora y d) 7 días.

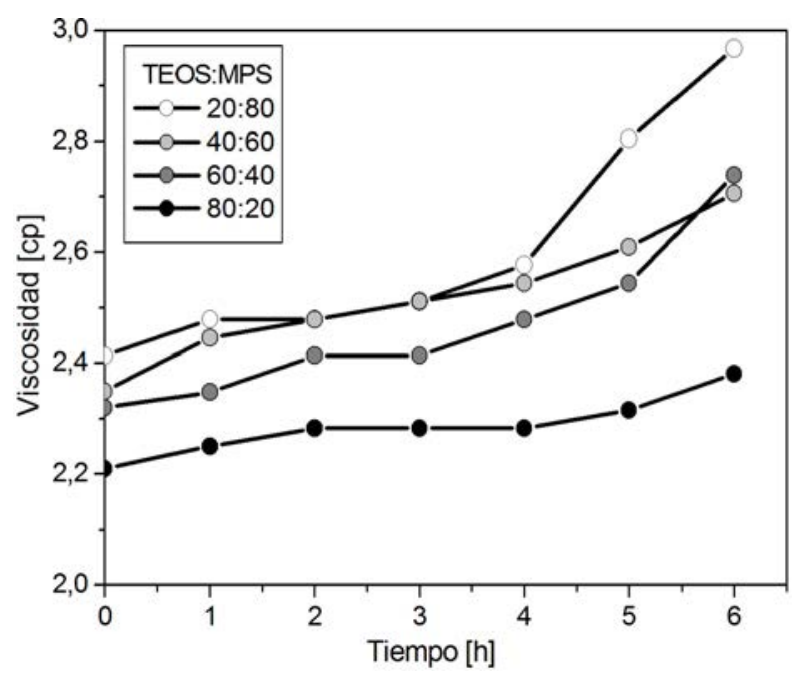

FIGURA 3. Evolución de la viscosidad de los soles TEOS:MPS para las relaciones molares estudiadas. recubrimientos, que favorece la formación de grupos silanol fundamentales para que se lleve a cabo la adecuada unión del recubrimiento con el sustrato metálico ( $\mathrm{Si}-\mathrm{O}-\mathrm{Al})$ permitiendo así una mejor mojabilidad del sol (Contreras et al., 2015).

Con relación al efecto de la relación TEOS/ MPS, en la Fig. 5 se muestran las micrografías electrónicas de los recubrimientos sintetizados para diferentes relaciones molares y $24 \mathrm{~h}$ de tiempo de envejecimiento del sol. En general, se observa que los recubrimientos no presentan agrietamiento o desprendimiento. Sin embargo, un alto contenido del precursor híbrido (20:80 40:60) conlleva a la obtención de recubrimientos con baja uniformidad y continuidad (Figs. 5a y $5 b)$. Por el contrario, el incremento del contenido del precursor inorgánico aumenta la mojabilidad del sol sobre el sustrato metálico, lo que se traduce en recubrimientos más homogéneos (Figs. 5c y 5d). 
(a)

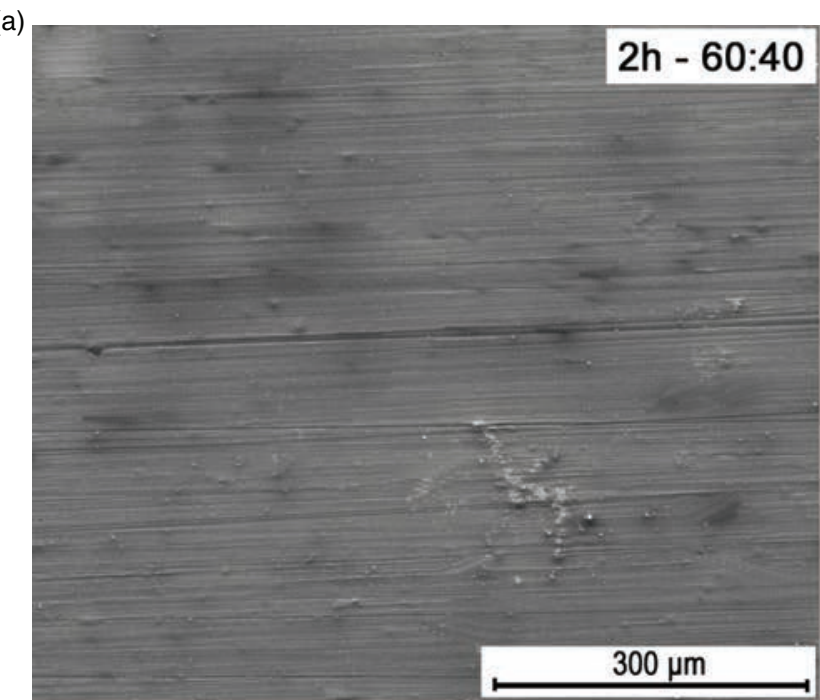

(c)

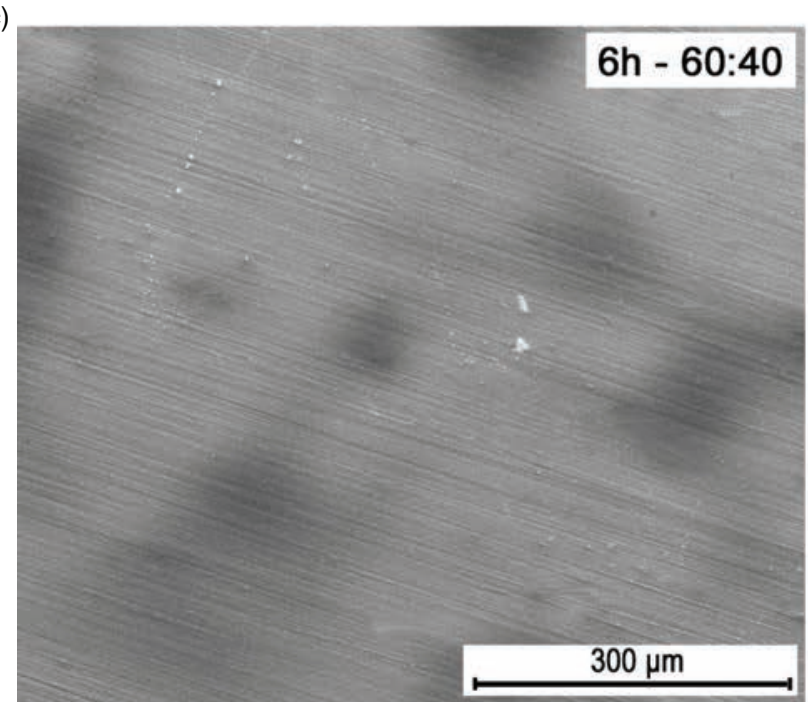

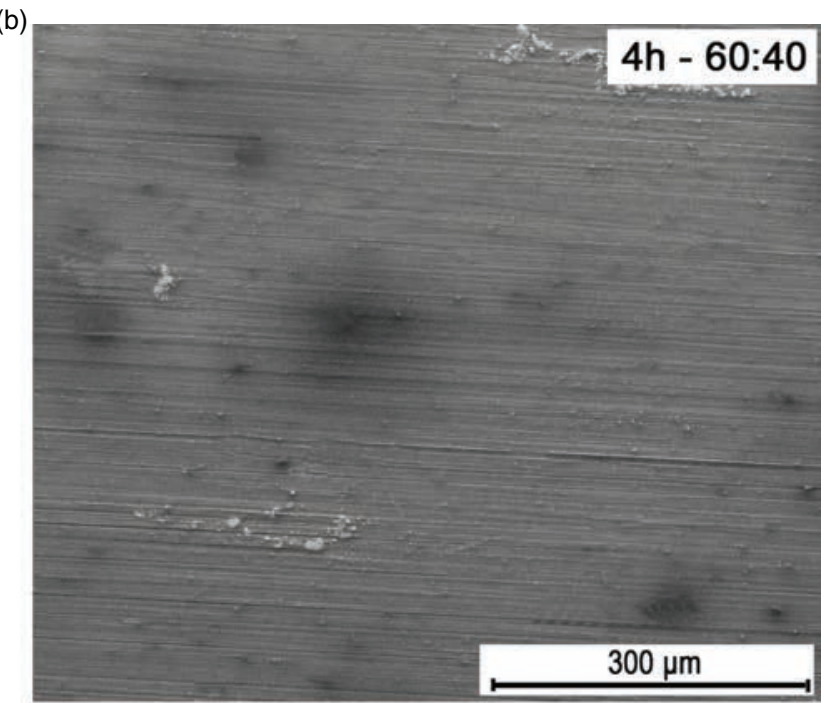

(d)

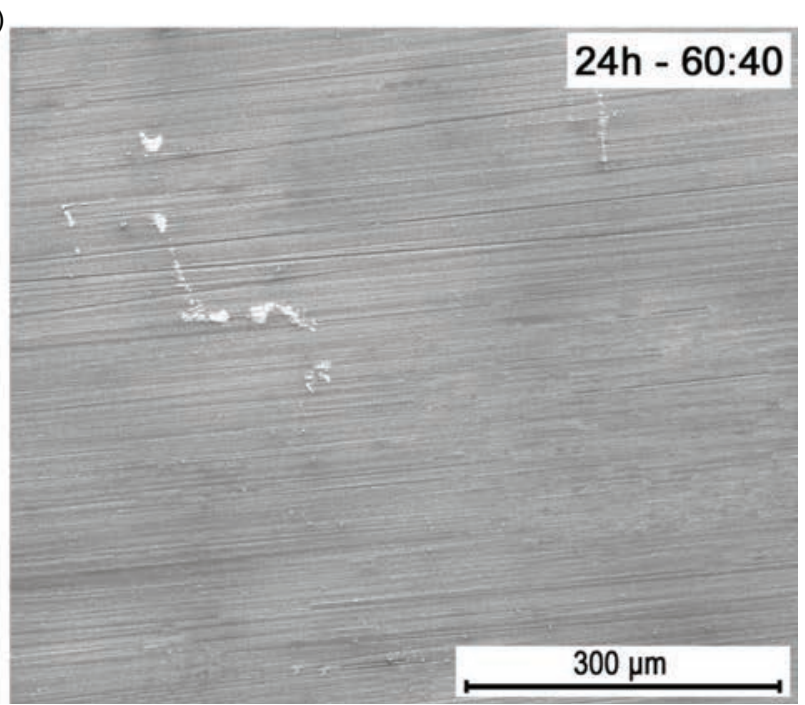

FIGURA 4. Micrografías electrónicas de los recubrimientos para la relación molar 60:40 TEOS:MPS y diferentes tiempos de envejecimiento.

Se puede apreciar en la Fig. 6 la influencia de cada uno de los precursores en la morfología de los recubrimientos, que generó dos efectos diferentes: i) los recubrimientos sintetizados con elevada relación molar de MPS presentaron una textura más lisa y plástica, similar a la que presentan los recubrimientos orgánicos amorfos (Fig. 6a); mientras que ii) los recubrimientos sintetizados con elevada relación molar de TEOS exhibieron una textura granular y cerámica característica de los recubrimientos inorgánicos, aunque sin la presencia del agrietamiento que los caracteriza (Fig. 6d).

De forma complementaria, en la Fig. 7 se presentan las micrografías electrónicas de la sección transversal de los recubrimientos sintetizados para las diferentes relaciones molares TEOS:MPS estudiadas a $24 \mathrm{~h}$ de envejecimiento de los soles. El análisis de dichas micrografías confirmó lo observado en las micrográficas superficiales, es decir, la formación de recubrimientos sin signos aparentes de agrietamiento y con buena adhesión al sustrato. No obstante, se observa una significativa diferencia de espesor de los recubrimientos con la variación de la relación molar de los precursores, de tal forma que para la mayor relación molar del precursor híbrido estudiada (20:80) se obtuvieron espesores de hasta $68 \mu \mathrm{m}$, aunque este espesor no es uniforme en toda la superficie, siendo posible 
(a)

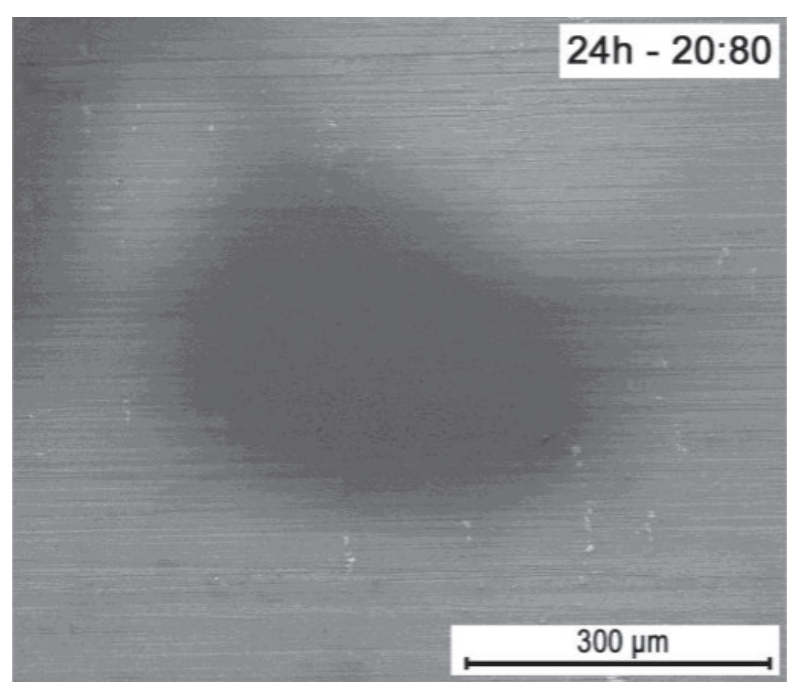

(c)

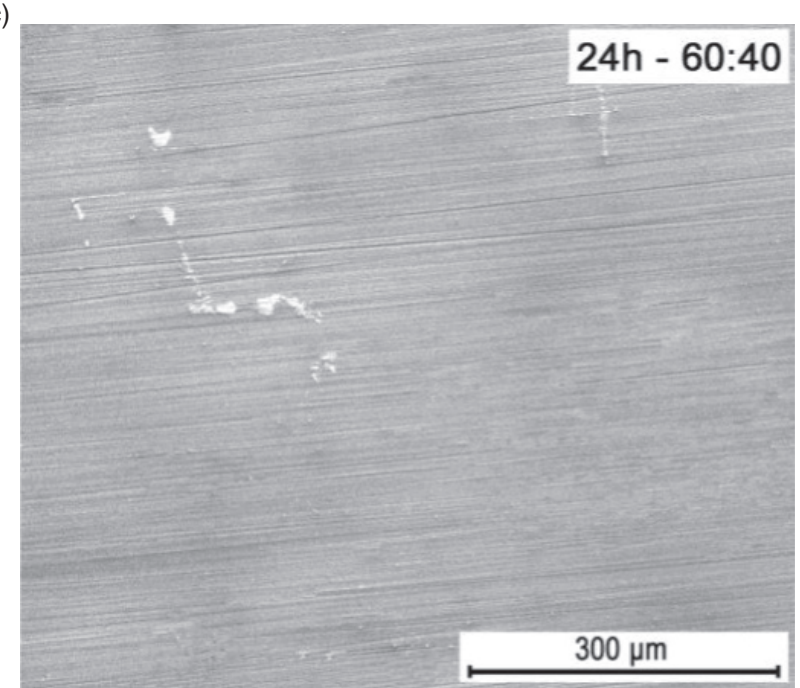

(b)

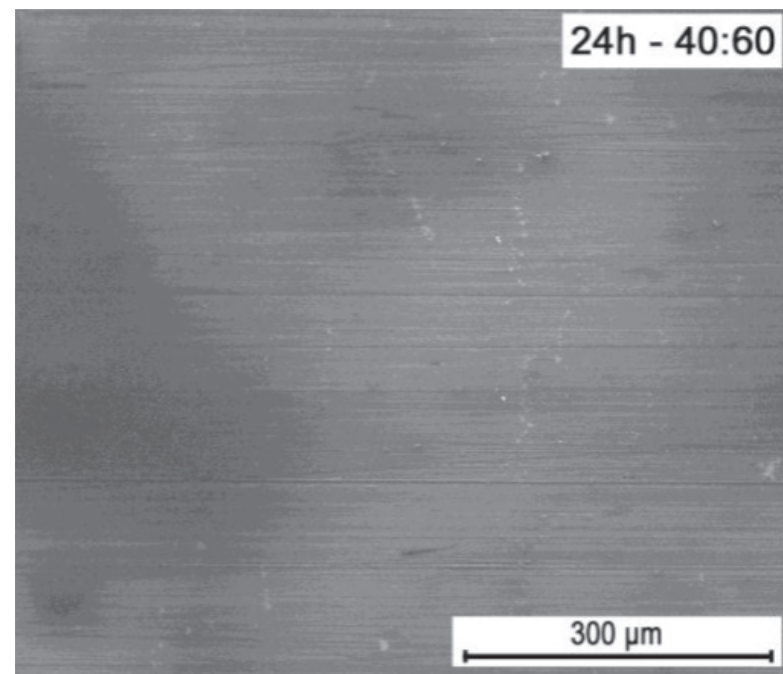

(d)

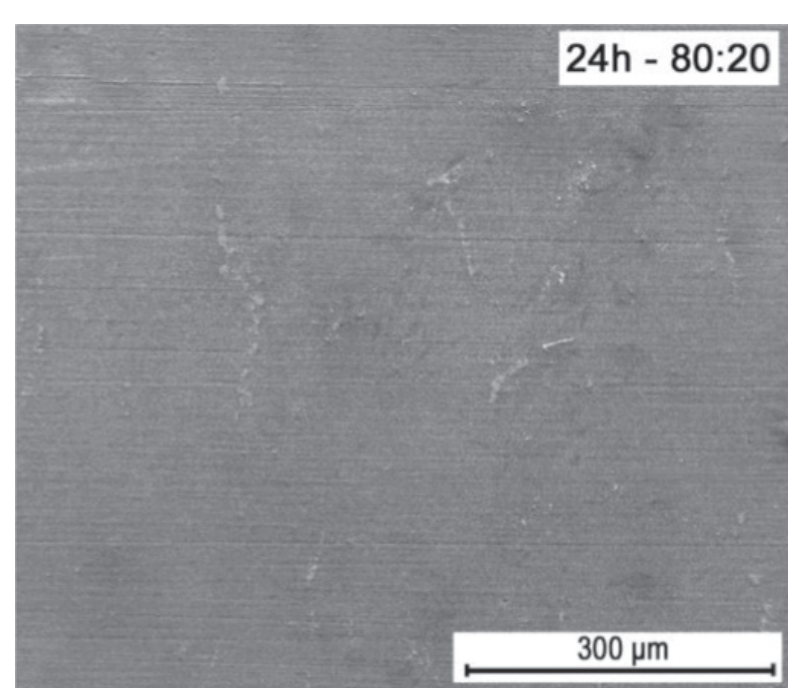

FIGURA 5. Micrografías electrónicas de los recubrimientos para diferentes relaciones molares TEOS:MPS a las 24 h de envejecimiento del sol.

observar espesores menores de $1 \mu \mathrm{m}$ en otras zonas de la muestra (Fig. 7a), dejando en evidencia una vez más la falta de uniformidad y continuidad de este tipo de recubrimientos. Por el contrario, el incremento del precursor inorgánico permite obtener recubrimientos con espesores entre 1 y $2 \mu \mathrm{m}$ aproximadamente, que son más continuos en toda la superficie del sustrato, favoreciendo la homogeneidad del recubrimiento, especialmente para la relación 80:20.

\subsection{Evaluación de la resistencia a la corrosión}

En la Fig. 8a se presentan las curvas de polarización anódica potenciodinámica de la aleación base y recubierta para la relación molar 20:80 a diferentes tiempos de envejecimiento del sol, después de ser expuestas en medio aireado de $0,1 \mathrm{M}$ de $\mathrm{NaCl}$ por un tiempo de $1 \mathrm{~h}$, a temperatura ambiente. En la curva de polarización de aleación AA2050-T8 se evidencia que este material no presenta potencial de picadura y experimenta corrosión de forma espontánea al ser expuesto en el medio agresivo. El potencial de corrosión se ubica en $-0,611 \mathrm{~V}$ y su densidad de corriente de corrosión $\left(i_{\text {corr }}\right)$ es $1,797 \mathrm{E}-7 \mathrm{~A} \cdot \mathrm{cm}^{-2}$. Por otra parte, con respecto a los recubrimientos sol-gel, se observa que, en general, para todos los tiempos de estudio, las películas sintetizadas mejoran la resistencia a la corrosión del material base, al disminuir los valores de $i_{\text {corr }}$ en alrededor de dos órdenes de magnitud y otorgar un rango de protección que, dependiendo de las condiciones de síntesis, alcanza valores superiores a $100 \mathrm{mV}$. 
(a)

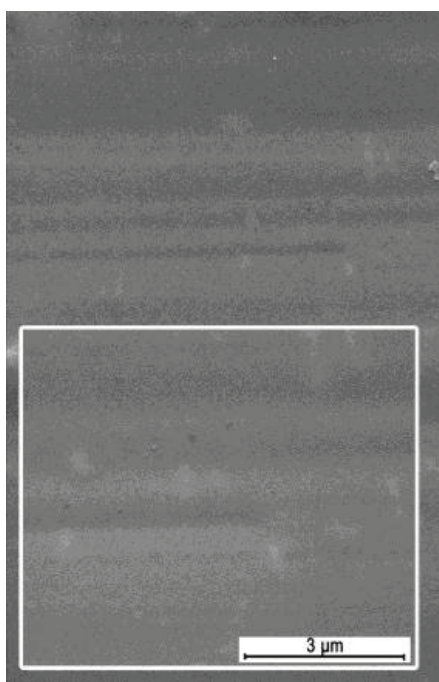

(c)

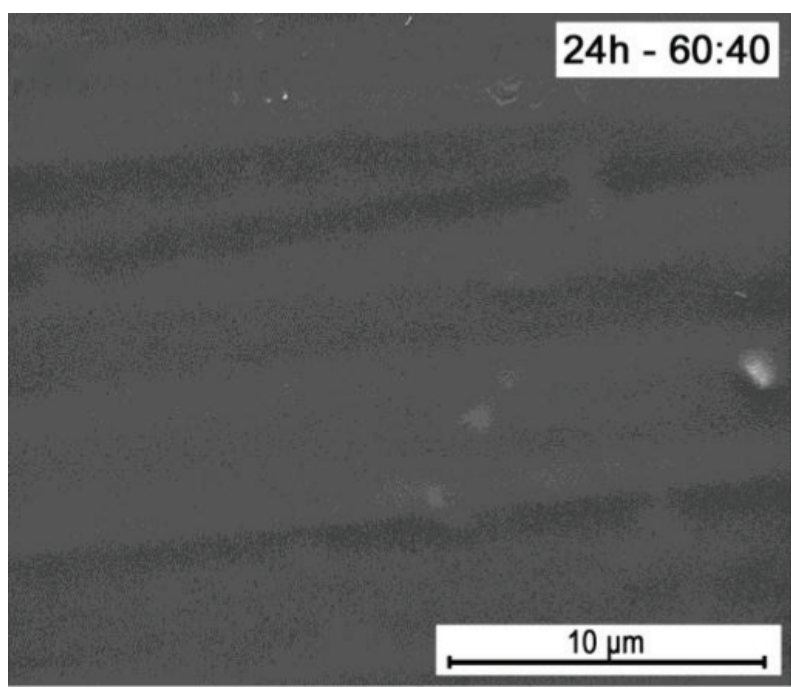

(b)

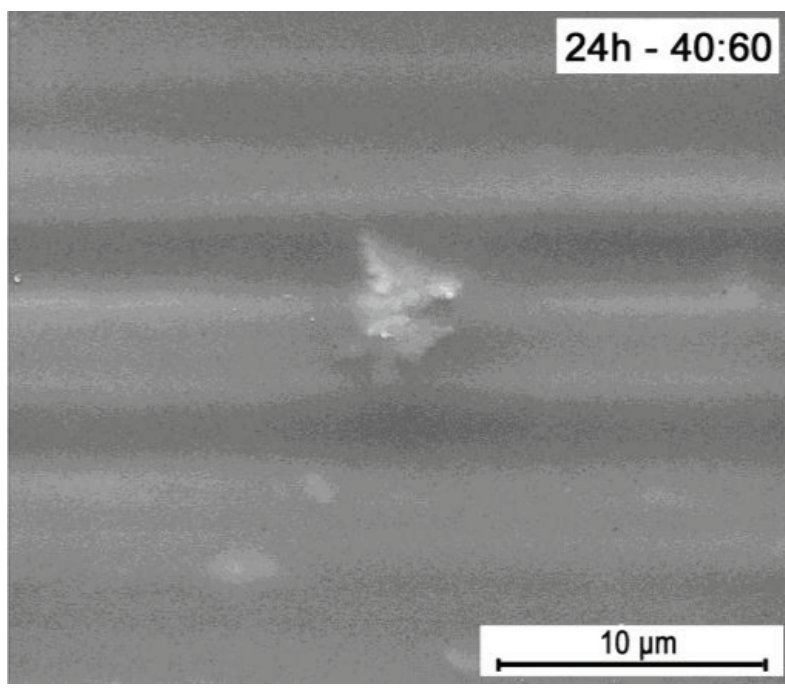

(d)

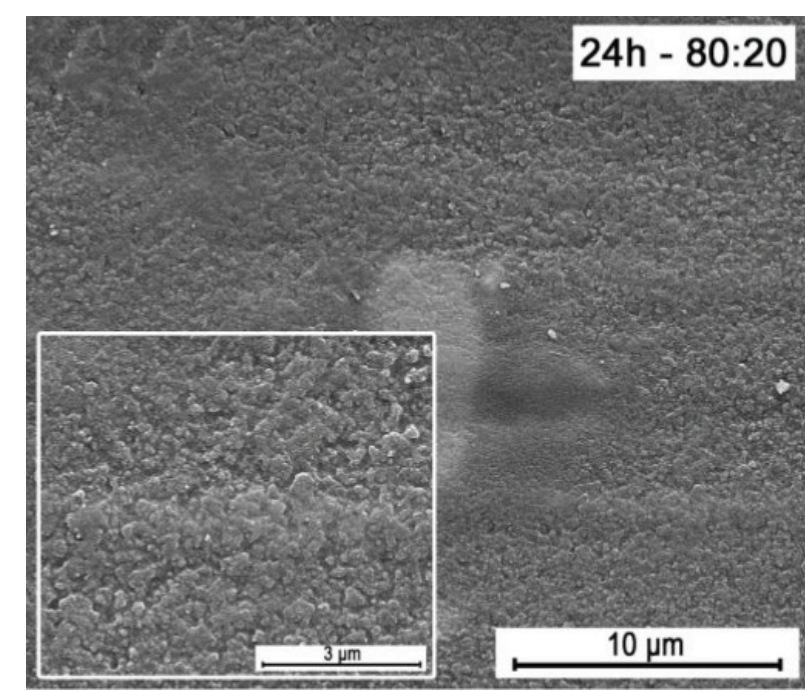

FIGURA 6. Micrografías electrónicas de los recubrimientos por sol-gel para diferentes relaciones molares TEOS:MPS.

Con respecto al efecto de la relación TEOS/ MPS, en la Fig. 8b se presentan las curvas de polarización anódica para las diferentes relaciones molares estudiadas a $4 \mathrm{~h}$ de envejecimiento del sol. Se aprecia que la relación molar TEOS/ MPS no afecta significativamente el valor de $\mathrm{i}_{\text {corr }}$, que se mantiene en el rango de $10^{-9}-10^{-10} \mathrm{~A} \cdot \mathrm{cm}^{-2}$; sin embargo, la relación 80:20 presenta una zona de pasivación más amplia $(\sim 0,170 \mathrm{~V})$, relacionada posiblemente con la mayor homogeneidad que exhiben los recubrimientos sintetizados bajo esta relación de precursores.

Finalmente, los parámetros electroquímicos más importantes de las curvas de polarización de los recubrimientos evaluados junto con los correspondientes al material base se reúnen en la Tabla 2.

\section{CONCLUSIONES}

- El etanol como solvente permite la estabilización y solubilización del precursor MPS al formar puentes de hidrógeno con el grupo carbonilo, y ser un medio adecuado para que se favorezca el proceso de polimerización de los grupos metacrilato en presencia de peróxido de benzoílo.

- El incremento de la relación molar del precursor inorgánico mejora significativamente las características morfológicas de los recubrimientos, especialmente en lo relacionado a uniformidad de espesor. Características que se ven favorecidas con el aumento del tiempo de envejecimiento del sol debido al mayor grado de hidrólisis. 
(a)

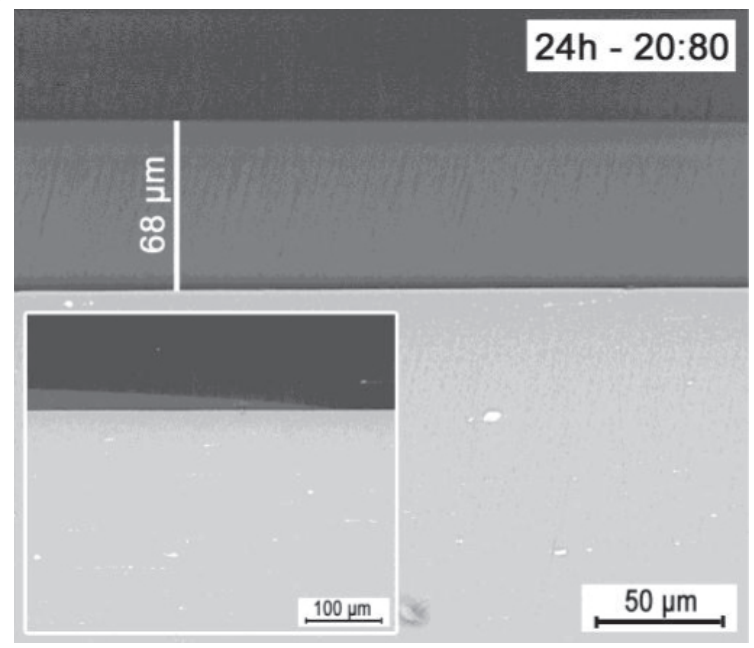

(c)

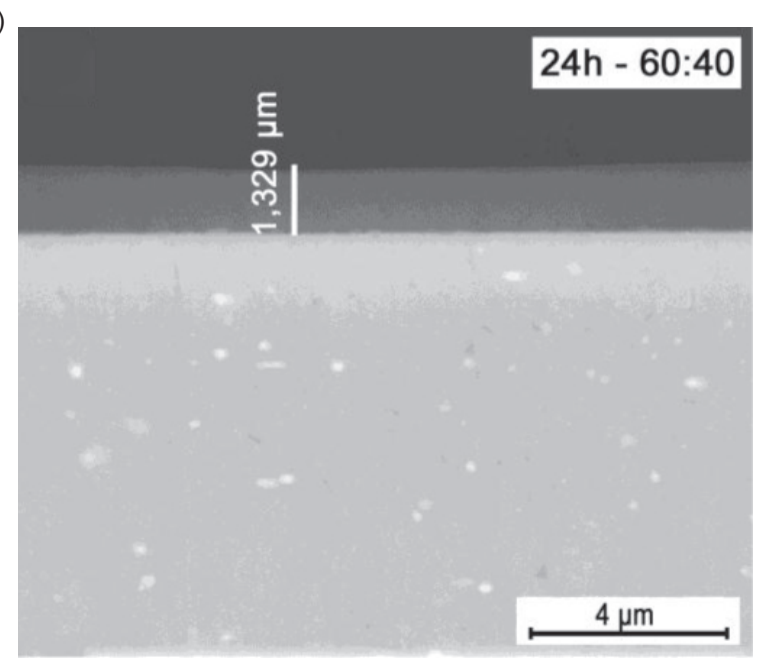

(b)

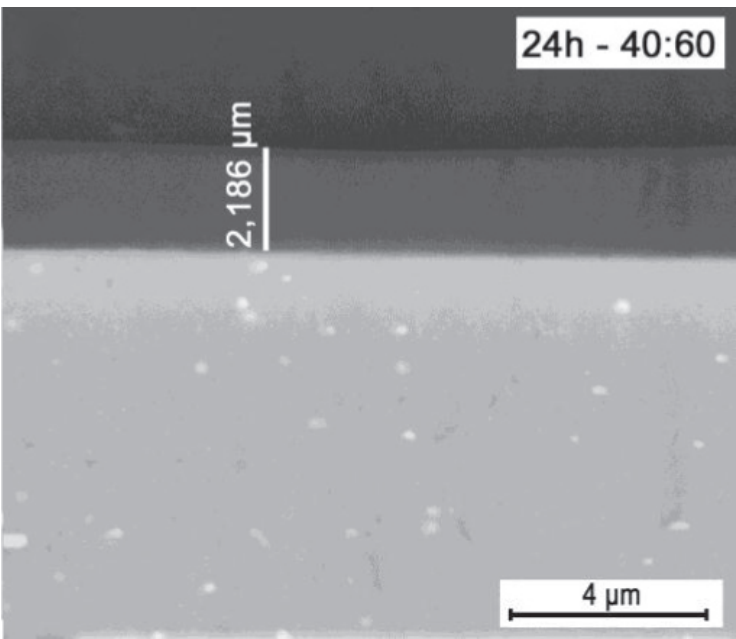

(d)

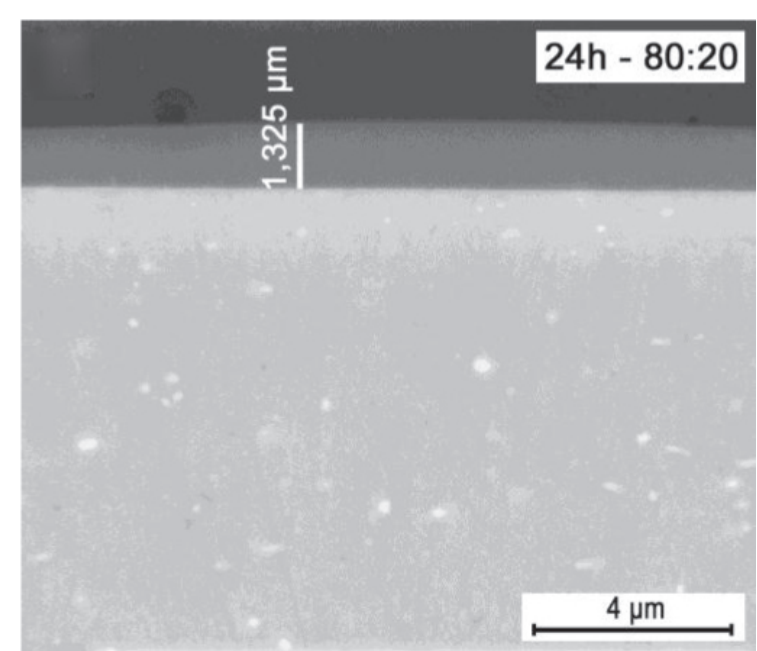

FiguRa 7. Micrografías electrónicas de la sección transversal de los recubrimientos a 24 h de envejecimiento y diferentes relaciones molares TEOS:MPS.

(a)

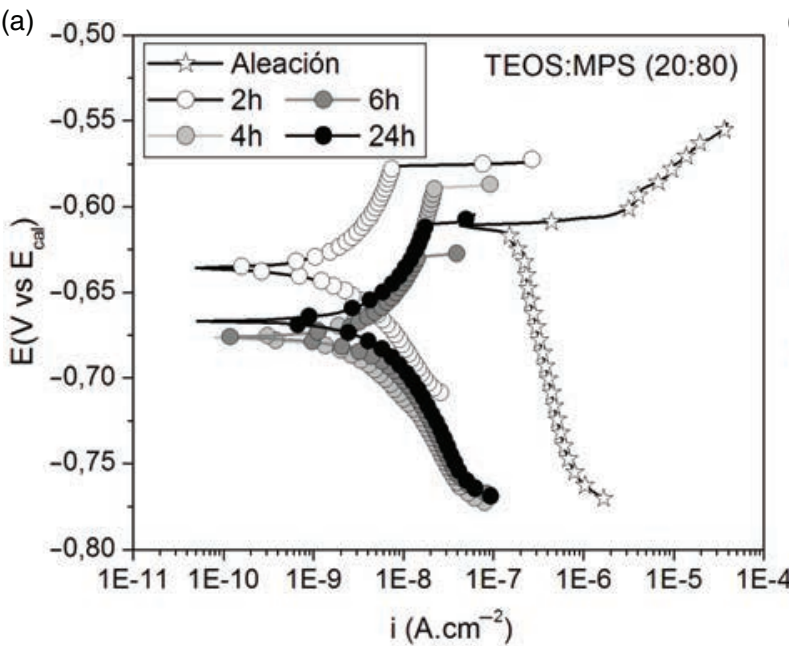

(b)

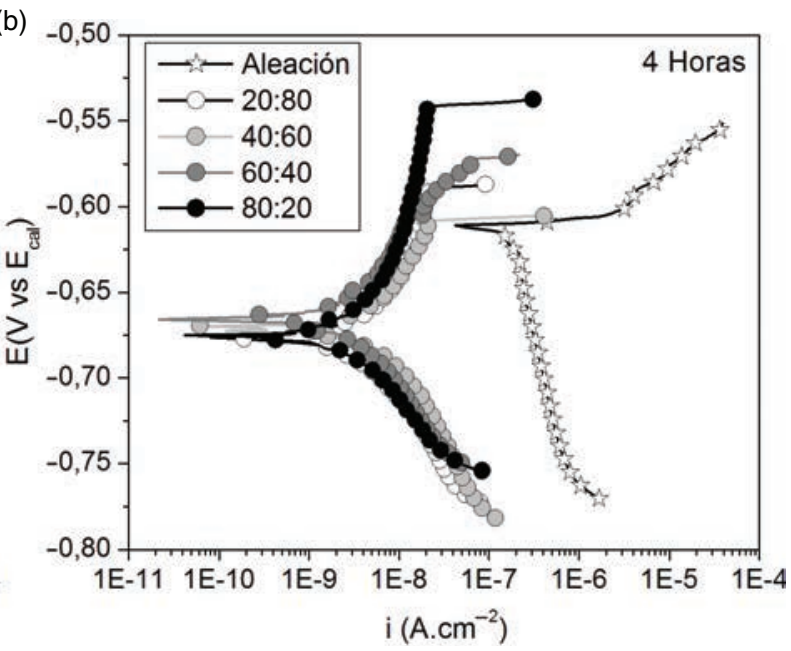

FIGURA 8. Curvas de polarización anódica potenciodinámica de la aleación base y recubierta: a) relación molar TEOS:MPS 20:80 y diferentes tiempos de envejecimiento del sol; b) 4 h de envejecimiento del sol y diferentes relaciones molares TEOS:MPS. 
Síntesis, caracterización y evaluación de la resistencia a la corrosión de recubrimientos híbridos Sol-Gel base TEOS/MPS • 11

TABLA 2. Parámetros electroquímicos de las curvas de polarización anódica de los recubrimientos evaluados

\begin{tabular}{|c|c|c|c|c|c|c|}
\hline $\begin{array}{l}\text { Relación Molar } \\
\text { TEOS:MPS }\end{array}$ & $\begin{array}{c}\text { Tiempo } \\
\text { Envejecimiento (h) }\end{array}$ & $E_{\text {corr }}(V)$ & $I_{\text {corr }}\left(A \cdot \mathrm{cm}^{-2}\right)$ & $\mathrm{E}_{\text {pic }}(\mathbf{V})$ & $E_{\text {pic }}-E_{\text {corr }}(V)$ & $I_{\text {pas }}\left(A \cdot \mathbf{c m}^{-2}\right)$ \\
\hline & AA2050-T8 & $-0,611$ & $1,797 \mathrm{E}-7$ & - & - & - \\
\hline \multirow[t]{4}{*}{ 20:80 } & 2 & $-0,635$ & $7,842 \mathrm{E}-10$ & $-0,575$ & 0,060 & $2,570 \mathrm{E}-9$ \\
\hline & 4 & $-0,675$ & $1,908 \mathrm{E}-9$ & $-0,587$ & 0,088 & $6,384 \mathrm{E}-9$ \\
\hline & 6 & $-0,676$ & $1,905 \mathrm{E}-9$ & $-0,627$ & 0,049 & $6,114 \mathrm{E}-9$ \\
\hline & 24 & $-0,665$ & $2,593 \mathrm{E}-9$ & $-0,608$ & 0,057 & $8,798 \mathrm{E}-9$ \\
\hline \multirow[t]{4}{*}{$40: 60$} & 2 & $-0,649$ & $1,297 \mathrm{E}-9$ & $-0,610$ & 0,039 & $4,839 \mathrm{E}-9$ \\
\hline & 4 & $-0,670$ & $2,325 \mathrm{E}-9$ & $-0,608$ & 0,062 & $7,740 \mathrm{E}-9$ \\
\hline & 6 & $-0,594$ & $1,628 \mathrm{E}-9$ & $-0,478$ & 0,116 & $2,553 \mathrm{E}-8$ \\
\hline & 24 & $-0,754$ & $9,599 \mathrm{E}-9$ & $-0,664$ & 0,090 & $4,725 \mathrm{E}-8$ \\
\hline \multirow[t]{4}{*}{$60: 40$} & 2 & $-0,617$ & $1,563 \mathrm{E}-9$ & $-0,575$ & 0,042 & $5,327 \mathrm{E}-9$ \\
\hline & 4 & $-0,665$ & $1,506 \mathrm{E}-9$ & $-0,571$ & 0,094 & $5,553 \mathrm{E}-9$ \\
\hline & 6 & $-0,586$ & $1,044 \mathrm{E}-9$ & $-0,559$ & $-0,027$ & $3,145 \mathrm{E}-9$ \\
\hline & 24 & $-0,636$ & $1,164 \mathrm{E}-9$ & $-0,543$ & 0,093 & $4,117 \mathrm{E}-9$ \\
\hline \multirow[t]{4}{*}{$80: 20$} & 2 & $-0,633$ & $9,219 \mathrm{E}-10$ & $-0,570$ & 0,063 & $4,032 \mathrm{E}-9$ \\
\hline & 4 & $-0,673$ & $9,566 \mathrm{E}-10$ & $-0,542$ & 0,131 & $5,625 \mathrm{E}-9$ \\
\hline & 6 & $-0,616$ & $6,856 \mathrm{E}-10$ & $-0,551$ & 0,065 & $3,054 \mathrm{E}-9$ \\
\hline & 24 & $-0,642$ & $1,721 \mathrm{E}-9$ & $-0,472$ & 0,170 & 7,425 E-9 \\
\hline
\end{tabular}

- En general, los recubrimientos sol-gel TEOS:MPS sintetizados sobre la aleación AA2050-T8 aumentaron su resistencia a la corrosión, al disminuir el valor de $\mathrm{i}_{\text {corr }}$ en dos órdenes de magnitud y otorgar un rango de pasivación de hasta $0,170 \mathrm{~V}$ aproximadamente, siendo este aumento más significativo para las películas sintetizadas con la relación molar 80:20.

\section{AGRADECIMIENTOS}

Los autores agradecen a la Vicerrectoría de Investigación y Extensión de la Universidad Industrial de Santander (Colombia) por la financiación del presente trabajo (Código de Proyecto 1880 y Programa para Movilidad de Estudiantes 2016). Así mismo, se agradece a la compañía Alcan Aerospace (Reino Unido) y a la Universidad de Manchester por proporcionar la aleación AA2050-T8 (Al-Cu-Li) para el desarrollo de la investigación.

\section{REFERENCIAS}

Barry Carte, C., Grant Norton, M. (2007). Sols, Gels, and Organic Chemistry, Ceramic materials science and engineering. Springer, Washington, pp. 400-411.

Boag, A., Hughes, A.E., Glenn, A.M., Muster, T.H., McCulloch, D. (2011). Corrosion of AA2024-T3 Part I: Localised corrosion of isolated IM particles. Corros. Sci. 53 (1), 17-26. http://dx.doi.org/10.1016/j. corsci.2010.09.009.
Bonekamp, B.C., Kreiter, R., Vente, J.F. (2008). Cap. Sol-gel approaches in the synthesis of membrane materials for nanofiltration and pervaporation. Sol-Gel methods for materials processing. Ed. Innocenzi, P., Zub, Y.L., Kessler, V.G., Published Springer, The Netherlands, pp. 47-65. https://link. springer.com/content/pdf/10.1007\%2F978-1-4020-8514-7. pdf.

Contreras, G., Nieves, C., Remolina, E., Hernández, C., Liu, Z., Coy, A., Viejo, F. (2015). Síntesis y evaluación de recubrimientos híbridos sol-gel base: TEOS: GPTMS: APTES para la protección contra la corrosión de la aleación AA2124-T4 y su material compuesto AA2124T4/25\%SiCp. Rev. LatinAm. Metal. Mat. 35, 222-236.

Coy, A.E., Viejo, F., Garcia-Garcia, F.J., Liu, Z., Skeldon, P., Thompson, G.E. (2010). Effect of excimer laser surface melting on the microstructure and corrosion performance of the die cast AZ91D magnesium alloy. Corros. Sci. 52 (2), 387-397. https://doi.org/10.1016/j.corsci.2009.09.025

Criado, M., Sobrados, I., Sanz, J. (2014). Polymerization of hybrid organic-inorganic materials from several silicon compounds followed by TGA/DTA, FTIR and NMR techniques. Prog. Org. Coat. 77, 880-891. http://dx.doi. org/10.1016/j.porgcoat.2014.01.019.

Crill, M.J., Chellman, D.J., Balmuth, E.S., Philbrook, M. Smith, K.P., Cho, A., Niedzinski, M., Muzzolini, R., Feiger, J. (2006). Evaluation of AA 2050-T87 Al-Li Alloy Crack Turning Behavior. Mater. Sci. Forum 519-521, 1323-1328. http://dx.doi.org/10.4028/www.scientific.net/ MSF.519-521.1323.

Embuka, D., Coy, A.E., Hernández-Barrios, C.A., Viejo, F., Liu, Z. (2017). Thermal stability of excimer laser melted films formed on the AA2024-T351 aluminium alloy: Microstructure and corrosion performance. Surf. Coat. Tech. 313, 214 221. https://dx.doi.org/10.1016/. surfcoat.2017.01.092.

Gharbi, O., Birbilis, N., Ogle, K. (2017). Li reactivity measurement during the surface pretreatment of $\mathrm{Al}-\mathrm{Li}$ alloy AA2050-T3. Electrochim. Acta 243, 207-219. https:// dx.doi.org/10.1016/j.electacta.2017.05.038.

Guérin, M., Alexis, J., Andrieu, E., Blanc, C., Odemer, G. (2015). Corrosion-fatigue lifetime of Aluminium-Copper-Lithium 
alloy 2050 in chloride solution. Mater. Design 87, 681-692. https://dx.doi.org/10.1016/j.matdes.2015.08.003.

Guo, R., Hu, C., Pan, F., Wu, H., Jiang, Z. (2006). PVAGPTMS/TEOS hybrid pervaporation membrane for dehydration of ethylene glycol aqueous solution. J. Membrane Sci. 281 (1-2), 454 462. http://dx.doi.org/10.1016/j. memsci.2006.04.015.

Han, Y.H., Taylor, A., Mantle, M.D., Knowles, K.M. (2007). Sol-gel-derived organic-inorganic hybrid materials. J. NonCryst. Solids. 353 (3), 313-320. http://dx.doi.org/10.1016/j. jnoncrysol.2006.05.042.

Hernández-Barrios, C.A., Remolina, E.N, Contreras, G.A., Parada, N.J., Coy, A.E., Viejo, F. (2014). Uso potencial de recubrimientos híbridos sol-gel como alternativa para la protección contra la corrosión de aleaciones ligeras. Rev. LatinAm. Metal. Mat. 34, 251-261.

Hofacker, S., Mechtel, M., Mager, M., Kraus, H. (2002). Sol-gel: a new tool for coatings chemistry. Prog. Org. Coat. 45 (2-3), 159-164. https://doi.org/10.1016/S0300-9440(02)00045-0.

Jiang, H., Zheng, Z., Wang, X. (2008). Kinetic study of methyltriethoxysilane (MTES) hydrolysis by FTIR spectroscopy under different temperatures and solvents. Vib. Spectrosc. 46 (1), 1-7. http://dx.doi.org/10.1016/j.vibspec. 2007.07.002.

Lequeu, P., Smith, K.P., Daniélou, A. (2010). Aluminum-Copper-Lithium Alloy 2050 Developed for Medium to Thick Plate. J. Mater. Eng. Perform. 19 (6), 841-847. http:// dx.doi.org/10.1007/s11665-009-9554-z.

Li, J.F, Zheng, Z.Q., Jiang, N., Li, S.C. (2005). Study on localized corrosion mechanism of $2195 \mathrm{Al}$-Li alloy in $4.0 \% \mathrm{NaCl}$ solution ( $\mathrm{pH}$ 6.5) using a three-electrode coupling system. Mater. Corros. 56 (3), 192-196. http://dx.doi.org/10.1002/ maco. 200403824

Li, J.F., Zheng, Z.Q., Li, S.C., Chen, W.J., Ren, W.D., Zhao, X.S. (2007). Simulation study on function mechanism of some precipitates in localized corrosion of $\mathrm{Al}$ alloys. Corros. Sci. 49 (6), 2436-2449. http://dx.doi.org/10.1016/j. corsci.2006.12.002.

Li, H.Y., Tang, Y., Zeng, Z., Zheng, F. (2008). Exfoliation corro-

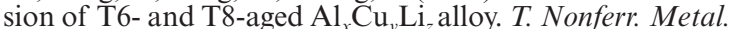
Soc. China 18 (4), 778-783. http://dx.doi.org/10.1016/ S1003-6326(08)60134-X.

Li, Y., Shi, Z., Lin, J., Yang, Y.L., Huang, B.M., Chung, T.F., Yang, J.R. (2016). Experimental investigation of tension and compression creep-ageing behaviour of AA2050 with different initial tempers. Mat. Sci. Eng. A-Struct. 657, 299308. http://dx.doi.org/10.1016/j.msea.2016.01.074.

Limpo, J., Rubio, J., Oteo, J.L. (1993). Estudio por FT-IR de la hidrólisis del tetraetilortosilicato. Bol. Soc. Esp. Ceram. V. 32 (1), 31-35. http://digital.csic.es/bitstream/10261/49490/1/bsecv-10-05-2012.pdf.

Liu, F., Shan, D., Han, E., Liu, C. (2008). Barium phosphate conversion coating on die-cast AZ91D magnesium alloy. T. Nonferr. Metal. Soc. China 18, 344-348. http://dx.doi. org/10.1016/S1003-6326(10)60229-4.

Ma, Y., Zhou, X., Thompson, G.E., Curioni, M., Zhong, X., Koroleva, E., Skeldon, P., Thomson, P., Fowles, M. (2011). Discontinuities in the porous anodic film formed on AA2099-T8 aluminium alloy. Corros. Sci. 53 (12), 41414151. http://dx.doi.org/10.1016/j.corsci.2011.08.023.

Malard, B., De Geuser, F., Deschamps, A. (2015). Microstructure distribution in an AA2050 T34 friction stir weld and its evolution during post-welding heat treatment. Acta Mater. 101, 90-100. http://dx.doi.org/10.1016/j. actamat.2015.08.068

Moulay, S. (2010). Chemical modification of poly (vinyl chloride) still on the run. Prog. Polym. Sci. 35 (3), 303-331. http://dx.doi.org/10.1016/j.progpolymsci.2009.12.001.

Ohtsuki, C., Miyazaki, T., Tanihara, M. (2002). Development of bioactive organic-inorganic hybrid for bone substitutes Mat. Sci. Eng. C 22 (1), 27-34. http://dx.doi.org/10.1016/ S0928-4931(02)00109-1.
Peña Alfonso, R., Rubio, F., Rubio, J., Oteo, J.L. (2007). Study of the hydrolysis and condensation of $\gamma$-Aminopropyltriethoxysilane by FT-IR spectroscopy. J. Mater. Sci. 42 (2), 595-603. http://dx.doi.org/10.1007/ s10853-006-1138-9.

Polmear, L. (2005). Light alloys: From traditional alloys to nanocrystals, Fourth Edition, Butterworth-Heinemann, Reino Unido.

Remolina, E.N, Hernández-Barrios, C.A., Coy, A.E., Viejo, F., Alba, N.C. (2017). Efecto de la adición de nitrato de cerio y el tiempo de envejecimiento en la síntesis de recubrimientos híbridos sol-gel base TEOS-GPTMS sobre la aleación de aluminio AA2050-T8. Rev. LatinAm. Metal. Mat. 37 (OnLine), http://www.rlmm.org/ojs/index.php/ rlmm/article/view/793.

Rosero, N.C., Pellice, S.A., Castro, Y., Aparicio, M., Durán, A. (2009). Improved corrosion resistance of AA2024 alloys through hybrid organic-inorganic sol-gel coatings produced from sols with controlled polymerization. Surf. Coat. Tech. 203 (13), 1897-1903. http://dx.doi.org/10.1016/j. surfcoat.2009.01.019.

Rosero, N.C., Paussa, L., Andreatta, F., Castro, Y., Durán, A., Aparicio, M., Fedrizzi, L. (2010a). Optimization of hybrid sol-gel coatings by combination of layers with complementary properties for corrosion protection of AA2024. Prog. Org. Coat. 69 (2), 167-174. http://dx.doi.org/10.1016/j. porgcoat.2010.04.013.

Rosero, N.C., Figiel, P., Jedrzejewski, R., Biedunkiewicz, A., Castro, Y., Aparicio, M., Pellice, S.A., Durán, A. (2010b). Influence of cerium concentration on the structure and properties of silica-methacrylate sol-gel coatings. J. SolGel Sci. Techn. 54 (3), 301-311. http://dx.doi.org/10.1007/ s10971-010-2194-9.

Rueda, L.M., Hernández-Barrios, C.A., Viejo, F., Coy, A.E., Mosa, J., Aparicio, M. (2016). Diseño de recubrimientos multicapa barrera-biomimético base TEOS-GPTMS sobre la aleación de magnesio Elektron 21 de potencial aplicación en la fabricación de implantes ortopédicos. Rev. Metal. 52 (3), e075. http://dx.doi.org/10.3989/revmetalm.075.

Téllez, L., Rubio, F. Peña-Alfonso, R., Rubio, J. (2004). Seguimiento por espectroscopia infrarroja (FT-IR) de la copolimerización de TEOS (tetraetilortosilicato) y PDMS (polidimetilsiloxano) en presencia de tbt (tetrabutiltitanio). Bol. Soc. Esp. Ceram. V. 43 (5), 883-890. http://digital.csic. es/bitstream/10261/4416/1/espectroscopia.pdf.

Viejo, F., Coy, A.E., Garcia-Garcia, F.J., Liu, Z., Skeldon, P., Thompson, G.E. (2010a). Relationship between microstructure and corrosion performance of AA2050-T8 aluminium alloy after excimer laser surface melting. Corros. Sci. 52 (6), 2179-2187. http://dx.doi.org/10.1016/j. corsci.2010.03.003.

Viejo, F., Aburas, Z., Coy, A.E., Garcia-Garcia, F.J., Liu, Z. Skeldon, P. Thompson, G.E. (2010b). Performance of Al alloys following excimer LSM - anodising approaches. Surf. Interface Anal. 42 (4), 252-257. http://dx.doi. org/10.1002/sia.3144

Wang, D., Bierwagen, G.P. (2009). Sol-gel coatings on metals for corrosion protection. Prog. Org. Coat. 64 (4), 327-338. http://dx.doi.org/10.1016/j.porgcoat.2008.08.010.

Wang, N., Wu, C., Wu Y., Xu, T. (2010). Hybrid anion exchange hollow fiber membranes through sol-gel process of different organic silanes within BPPO matrix. J. Membrane Sci. 363 (1-2), 128-139. http://dx.doi.org/10.1016/j. memsci. 2010.07.020.

Warner, T. (2006). Recently-developed aluminium solutions for aerospace applications. Mater. Sci. Forum. 519-521, 1271-1278. http://dx.doi.org/10.4028/www.scientific.net/ MSF.519-521.1271

Zheng, S., Li, J. (2010). Inorganic-organic sol gel hybrid coatings for corrosion protection of metals. J. Sol-Gel Sci. Techn. 54 (2), 174-187. http://dx.doi.org/10.1007/ s10971-010-2173-1. 